PROCEEDINGS OF THE

AMERICAN MATHEMATICAL SOCIETY

Volume 130, Number 2, Pages 315-325

S 0002-9939(01)06087-7

Article electronically published on June 19, 2001

\title{
$S L_{2}$ ACTION ON THE COHOMOLOGY OF A RANK TWO ABELIAN GROUP WITH ARBITRARY COEFFICIENT DOMAIN
}

\author{
ERIC JESPERS AND ALEXANDER ZIMMERMANN
}

(Communicated by Stephen D. Smith)

\begin{abstract}
A rank two abelian group $C_{n} \times C_{n}$ is in a natural way an $S L_{2}(\mathbb{Z})$ module. This induces an action of $S L_{2}(\mathbb{Z})$ on its group cohomology $H^{m}\left(C_{n} \times C_{n}, R\right)$ for any trivial coefficient domain $R$. In the present note we determine this module, including the question of when the universal coefficient theorem sequence splits.
\end{abstract}

\section{INTRODUCTION}

Let $G$ be a group and $R$ a commutative ring, seen as a trivial $R G$-module. Then, $\operatorname{Aut}(G)$, the automorphism group acts on $G$, but also, $R$-linearly, on $H^{m}(G, R)$, the group cohomology in degree $m$ for any $m \in \mathbb{N}$. The universal coefficient theorem (e.g. [1, Theorem 2.7.1], with the appropriate modification made since we deal with cochain complexes) gives a natural exact sequence

$$
0 \longrightarrow H^{m}\left(C_{n}^{2}, \mathbb{Z}\right) \otimes_{\mathbb{Z}} R \longrightarrow H^{m}\left(C_{n}^{2}, R\right) \longrightarrow \operatorname{Tor}_{1}^{\mathbb{Z}}\left(R, H^{m+1}\left(C_{n}^{2}, \mathbb{Z}\right)\right) \longrightarrow 0 .
$$

So, once the module structure of $H^{m}\left(C_{n}^{2}, \mathbb{Z}\right)$ is known, one gets the module structure of $H^{m}\left(C_{n}^{2}, R\right)$ for any coefficient domain $R$, up to the nature of an exact sequence. Moreover, for any group $G$ the Bockstein sequence gives an exact sequence

$$
\ldots \longrightarrow H^{m}(G, \mathbb{Z}) \stackrel{k \cdot}{\longrightarrow} H^{m}(G, \mathbb{Z}) \longrightarrow H^{m}(G, \mathbb{Z} / k \mathbb{Z}) \longrightarrow H^{m+1}(G, \mathbb{Z}) \longrightarrow \ldots
$$

and in case $G$ is an abelian group of exponent $n=k$, this yields an exact sequence

$$
0 \longrightarrow H^{m}(G, \mathbb{Z}) \longrightarrow H^{m}(G, \mathbb{Z} / n \mathbb{Z}) \longrightarrow H^{m+1}(G, \mathbb{Z}) \longrightarrow 0
$$

In case $G$ is an elementary abelian $p$-group of rank $\rho$ and $n=p$ for an odd prime $p$, the $G L_{\rho}(\mathbb{Z})$-module $H^{m}(G, \mathbb{Z} / n \mathbb{Z})$ is clearly the $m$-th homogeneous component of the tensor product of the symmetric and the exterior algebra on the natural representation, where the generators of the exterior algebra are in degree 1 and the generators of the symmetric algebra are in degree 2 . The case $p=2$ is similar; only the exterior algebra does not occur and the generators of the symmetric algebra are in degree 1. This is an immediate consequence of the well known structure of the $\bmod p$ cohomology rings of these groups. Chapman [2] also gives the integral

Received by the editors May 19, 2000 and, in revised form, June 12, 2000.

2000 Mathematics Subject Classification. Primary 20J06, 20C05, 20F29.

This research was done while the authors collaborated at the "Mathematisches Forschungsinstitut Oberwolfach" financed by the "Research in Pairs" program of the "Volkswagen Stiftung". The first-named author is also supported in part by Fonds voor Wetenschappelijk Ondezoek (Belgium) and Onderzoeksraad Vrije Universiteit Brussel. 
cohomology of an abelian group. Nevertheless, the structure there is much less appealing, even more as his results are valid for odd groups only.

The above sequences give a recursive understanding of the composition factors of $H^{m}\left(C_{n}^{\rho}, R\right)$ as an $R G L_{\rho}(\mathbb{Z})$-module. It does not tell much about how these factors are glued together. Using elementary methods, in the present article we try to close this gap and give the entire module structure for $\rho=2$.

Theorem 1. Let $n$ be a natural number and let $R$ be a commutative ring regarded as a trivial $C_{n}^{2}$-module. Let $S_{k}(A)$ be the symmetric algebra of degree $k$ over the free $A$-module $A^{2}$. The group $S l_{2}(\mathbb{Z})$ acts on this algebra in the natural way. Let $\Gamma(2)$ be the level 2 congruence subgroup of $S L_{2}(\mathbb{Z})$. Let ${ }_{n} R:=\{r \in R \mid n r=0\}$ be the ideal of $n$-torsion elements in $R$. Then, as $R S L_{2}(\mathbb{Z})$-modules we have the following exact sequences:

$$
0 \longrightarrow S_{k}(R / n R) \longrightarrow H^{2 k}\left(C_{n} \times C_{n}, R\right) \longrightarrow{ }_{n} R \cdot S_{k-1}(R) \longrightarrow 0
$$

for $k \geq 1$. This sequence is non-split exactly if $\left(\begin{array}{l}n \\ 2\end{array}\right) \cdot{ }_{n} R \nsubseteq n R$. The sequence regarded as $R \Gamma(2)$-modules is always split.

Moreover, we have an exact sequence

$$
0 \longrightarrow S_{k-1}(R / n R) \longrightarrow H^{2 k+1}\left(C_{n} \times C_{n}, R\right) \longrightarrow{ }_{n} R \cdot S_{k+1}(R) \longrightarrow 0
$$

for $k \geq 1$.

We remark that in case $n$ is odd, then $\left(\begin{array}{l}n \\ 2\end{array}\right) \cdot{ }_{n} R \subseteq n R$. In case $n$ is even, then this condition may or may not be satisfied. For example, for $R=\mathbb{Z} / 2^{\nu} \mathbb{Z}$ and $n=2^{\ell}$, one gets that the exact sequence coming from the universal coefficient theorem in even degree is split if and only if $\nu \neq \ell$.

The paper is organized as follows. In section 1 we introduce a particular sign convention for the tensor product of two projective resolutions of cyclic groups. Section 2 determines the lifting of the identity map to the projective resolutions obtained by twisting with the generator by the standard Borel subgroup. In section 3 the same is done for the Levi complement. Then, in section 4 we determine an adapted basis for the group cohomology. In section 4 the final section, we prove the theorem and discuss the splitting question.

\section{Setup}

Let $C_{n}$ be the cyclic group of order $n$. In this section we set up the notation to determine the action of $S l_{2}(\mathbb{Z})$ on $H^{*}\left(C_{n} \times C_{n}, \mathbb{Z}\right)$ induced by the natural action of $S l_{2}(\mathbb{Z})$ on $C_{n} \times C_{n}$. Of course, the action will have $\Gamma(n)$, the congruence subgroup of level $n$ in the kernel. So, in fact, we shall construct an action of $S l_{2}(\mathbb{Z} / n \mathbb{Z})$. But, as we shall see, this will only formally depend on $n$.

First we have to get a projective resolution of $\mathbb{Z}$ as a $\mathbb{Z}\left(C_{n} \times C_{n}\right)$-module. Let $\Gamma=\mathbb{Z}\left(C_{n} \times C_{n}\right)$. A free resolution $P$ of $\mathbb{Z}$ as a $\mathbb{Z}\left(C_{n} \times C_{n}\right)$-module is given by the tensor product of two copies of the ordinary free resolutions of $\mathbb{Z}$ as a $\mathbb{Z} C_{n}$-module. We see that the homogeneous component in degree $m$ is

$$
P_{m}:=\Gamma^{m+1}:=\bigoplus_{r=1}^{m+1} \Gamma .
$$

Write

$$
C_{n}^{2}=\left\langle a, b \mid a^{n}=b^{n}=a b a^{-1} b^{-1}=1\right\rangle
$$


and set $B:=b-1$ and $A:=a-1$. Moreover, $\Delta_{a}:=\sum_{i=1}^{n} a^{i}, \Delta_{a b}:=\sum_{i=1}^{n}(a b)^{i}$ and $\Delta_{b}:=\sum_{i=1}^{n} b^{i}$. We abbreviate

$$
J:=\left(\begin{array}{cc}
0 & \Delta_{a} \\
0 & 0
\end{array}\right), K:=\left(\begin{array}{cc}
\Delta_{b} & 0 \\
-A & B
\end{array}\right) \text { and } D:=\left(\begin{array}{cc}
B & 0 \\
A & \Delta_{b}
\end{array}\right) .
$$

The differential $d$ in degree $n$ is then multiplication from the right by the following matrices:

$$
D_{2 m+1}=\left(\begin{array}{ccccc}
K & 0 & 0 & \cdots & 0 \\
J & K & 0 & & \vdots \\
0 & J & K & \ddots & \vdots \\
\vdots & \ddots & \ddots & \ddots & 0 \\
0 & \ldots & 0 & J & K \\
0 & \cdots & \cdots & 0 & \left(0, \Delta_{a}\right)
\end{array}\right)_{(m+2, m+1)}
$$

and

$$
D_{2 m}=\left(\begin{array}{cccccc}
D & 0 & 0 & \cdots & 0 & 0 \\
-J & D & 0 & & \vdots & \vdots \\
0 & -J & D & \ddots & \vdots & \vdots \\
\vdots & \ddots & \ddots & \ddots & 0 & 0 \\
0 & \ldots & 0 & -J & D & 0 \\
0 & \ldots & \ldots & 0 & -J & (B \\
A
\end{array}\right)_{(m+1, m+2)}
$$

Thus $d_{n}: \Gamma^{n+2} \rightarrow \Gamma^{n+1}$.

We shall define an action of $S l_{2}(\mathbb{Z})$ on $H^{*}\left(C_{n}^{2}, \mathbb{Z}\right)$. Generating elements for $S l_{2}(\mathbb{Z})$ are $\alpha:=\left(\begin{array}{ll}1 & 1 \\ 0 & 1\end{array}\right)$ and $\beta:=\left(\begin{array}{cc}0 & -1 \\ 1 & 0\end{array}\right)$. This means $\alpha(a)=a, \alpha(b)=a b, \beta(a)=b$ and $\beta(b)=a^{-1}$. Clearly, $\alpha \beta$ has order 6 and $\beta$ has order 4 . What we have to find are chain isotopies $\tau_{\alpha}$ and $\tau_{\beta}$ such that

$$
\tau_{\alpha}: P \stackrel{\sim}{\longrightarrow}{ }^{\alpha} P \text { and } \tau_{\beta}: P \stackrel{\sim}{\longrightarrow}{ }^{\beta} P
$$

are isomorphisms as $\mathbb{Z} G$-complexes.

\section{The Borel SUbGroup ACtion}

We need some notation. Set $M:=\left(\begin{array}{ll}1 & b \\ 0 & 1\end{array}\right)$ and $N:=\left(\begin{array}{ll}1 & \delta \\ 0 & 1\end{array}\right)$ for

$$
\delta:=-\sum_{i=1}^{n-1} b^{i} \sum_{j=0}^{i-1} a^{j} \in \mathbb{Z}\left(C_{n} \times C_{n}\right) .
$$

Define matrices $T_{2 k}$ and $T_{2 k+1}$ by

$$
\left(T_{2 k+1}\right)_{i, j}=\left\{\begin{array}{cc}
0 & \text { if } k+1 \geq i>j, \\
\left(\begin{array}{l}
j-1 \\
i-1
\end{array}\right) M & \text { if } i \leq j \leq k+1
\end{array}\right.
$$


and

$$
\left(T_{2 k}\right)_{i, j}=\left\{\begin{array}{cc}
0 & \text { if } i>j \\
\left(\begin{array}{c}
j-1 \\
i-1
\end{array}\right) N & \text { if } i \leq j \leq k \\
\left(\begin{array}{c}
k \\
i-1
\end{array}\right) \cdot\left(\begin{array}{c}
1 \\
0
\end{array}\right) & \text { if } i<j=k+1 \\
1 & \text { if } i=j=k+1
\end{array}\right.
$$

\section{Lemma 1.}

$$
T_{k+1} \cdot D_{k}=\alpha\left(D_{k}\right) \cdot T_{k} \text { for any } k \in \mathbb{N} .
$$

Proof. We proceed by induction on $k$.

In degree 0 , the statement is true, since there the matrix $T$ is the identity matrix.

For the induction step we use the following identities:

$\delta \cdot A=\Delta_{b}-\Delta_{a b}$ and $\delta \cdot B=-\Delta_{a}+b \Delta_{a b}$ as well as $\delta \cdot(a b-1)=\Delta_{b}-\Delta_{a}$.

Now observe

$$
\begin{aligned}
u \cdot \alpha & (D) N+v \cdot \alpha(J) N \\
& =u\left(\begin{array}{cc}
a b-1 & 0 \\
a-1 & \Delta_{a b}
\end{array}\right) \cdot\left(\begin{array}{ll}
1 & \delta \\
0 & 1
\end{array}\right)+v \cdot\left(\begin{array}{cc}
0 & \Delta_{a} \\
0 & 0
\end{array}\right) \cdot\left(\begin{array}{cc}
1 & \delta \\
0 & 1
\end{array}\right) \\
& =u\left(\begin{array}{cc}
a b-1 & \Delta_{b} \\
A & \Delta_{b}
\end{array}\right)+(v-u)\left(\begin{array}{cc}
0 & \Delta_{a} \\
0 & 0
\end{array}\right) \\
& =u\left(\begin{array}{cc}
1 & b \\
0 & 1
\end{array}\right) \cdot\left(\begin{array}{cc}
B & 0 \\
A & \Delta_{b}
\end{array}\right)+(v-u)\left(\begin{array}{cc}
1 & b \\
0 & 1
\end{array}\right) \cdot\left(\begin{array}{cc}
0 & \Delta_{a} \\
0 & 0
\end{array}\right) \\
& =u M D+(v-u) M J .
\end{aligned}
$$

By similar computations we get $x \cdot J+y \cdot \alpha(K) M=(x+y) \cdot J+y \cdot N K$. Now,

$$
\begin{aligned}
&\left(T_{2 k+1} \cdot D_{2 k}\right)_{i, j}=\left\{\begin{array}{cc}
\left(\begin{array}{c}
j-1 \\
i-1
\end{array}\right) M D-\left(\begin{array}{c}
j \\
i-1
\end{array}\right) M J & \text { if } k+1>j \geq i, \\
\left(\begin{array}{c}
k \\
i-1
\end{array}\right) M\left(\begin{array}{c}
B \\
A
\end{array}\right) & \text { if } k+1=j \geq i, \\
-J & \text { if } j=i-1,
\end{array}\right. \\
&=\left\{\begin{array}{cc}
\text { if } j<i-1, \\
0 & \text { if } k+1>j \geq i,
\end{array}\right. \\
&\left(\begin{array}{cc}
\left(\begin{array}{c}
j-1 \\
i-1
\end{array}\right) M D-\left(\begin{array}{c}
j \\
i-1
\end{array}\right) M J \\
i-1)\left(\begin{array}{c}
a b-1 \\
A
\end{array}\right) & \text { if } k+1=j \geq i, \\
-J & \text { if } j=i-1, \\
0 & \text { if } j<i-1 .
\end{array}\right.
\end{aligned}
$$

On the other hand,

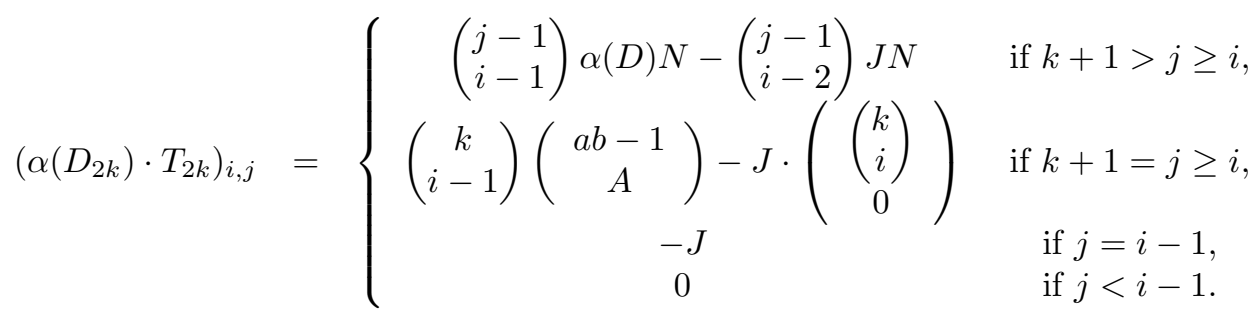


Because $M D-M J=\alpha(D) N$ and $M J=J N$ we get

$$
\begin{aligned}
\left(\begin{array}{l}
j-1 \\
i-1
\end{array}\right) \alpha(D) N-\left(\begin{array}{l}
j-1 \\
i-2
\end{array}\right) J N & =\left(\begin{array}{l}
j-1 \\
i-1
\end{array}\right) M D+\left(-\left(\begin{array}{l}
j-1 \\
i-2
\end{array}\right)-\left(\begin{array}{l}
j-1 \\
i-1
\end{array}\right)\right) M J \\
& =\left(\begin{array}{l}
j-1 \\
i-1
\end{array}\right) M D-\left(\begin{array}{c}
j \\
i-1
\end{array}\right) M J .
\end{aligned}
$$

As $J \cdot\left(\begin{array}{c}k \\ i \\ 0\end{array}\right)=\left(\begin{array}{l}0 \\ 0\end{array}\right)$ we thus get $T_{2 k+1} D_{2 k}=\alpha\left(D_{2 k}\right) T_{2 k}$.

We still have to prove $T_{2 k+2} \cdot D_{2 k+1}=\alpha\left(D_{2 k+1}\right) \cdot T_{2 k+1}$. Since $N J=J$ we get

$$
\left(T_{2 k+2} \cdot D_{2 k+1}\right)_{i, j}=\left\{\begin{array}{cc}
\text { if } i>j+1, \\
J & \text { if } i=j+1<k+1, \\
\left(\begin{array}{c}
j-1 \\
i-1
\end{array}\right) N K+\left(\begin{array}{c}
j \\
i-1
\end{array}\right) J & \text { if } i<j+1 \leq k+1, \\
\left(0 \Delta_{a}\right) & \text { if } i=k+1=j+1,
\end{array}\right.
$$

and, because $J M=J$ and $\left(0 \Delta_{a}\right) M=\left(0 \Delta_{a}\right)$, we obtain

$$
\left(\alpha\left(D_{2 k+1}\right) \cdot T_{2 k+1}\right)_{i, j}=\left\{\begin{array}{cc}
0 & \text { if } i>j+1 \\
J & \text { if } i=j+1<k+1, \\
\left(\begin{array}{l}
j-1 \\
i-1
\end{array}\right) \alpha(K) M+\left(\begin{array}{l}
j-1 \\
i-2
\end{array}\right) J & \text { if } i<j+1 \leq k+1 \\
\left(0 \Delta_{a}\right) & \text { if } i=k+1=j+1 .
\end{array}\right.
$$

Now, $\alpha(K) M=N K+J$ and therefore

$$
\begin{aligned}
\left(\begin{array}{l}
j-1 \\
i-1
\end{array}\right) \alpha(K) M+\left(\begin{array}{l}
j-1 \\
i-2
\end{array}\right) J & =\left(\begin{array}{l}
j-1 \\
i-1
\end{array}\right) N K+\left(\left(\begin{array}{c}
j-1 \\
i-1
\end{array}\right)+\left(\begin{array}{l}
j-1 \\
i-2
\end{array}\right)\right) J \\
& =\left(\begin{array}{l}
j-1 \\
i-1
\end{array}\right) N K+\left(\begin{array}{c}
j \\
i-1
\end{array}\right) J .
\end{aligned}
$$

Thus the result follows.

Lemma 2. Define

$$
\tau_{k-1}(x):=\alpha(x) \cdot T_{k}
$$

Then, these mappings $\tau$ define a chain isomorphism $P \longrightarrow{ }^{\alpha} P$.

Proof. This follows from Lemma 1 In fact, the so-defined mappings make the following diagrams commutative:

$$
\begin{aligned}
& \Gamma^{2 n+1} \stackrel{\cdot D_{2 n}}{\longleftarrow} \quad \Gamma^{2 n+2} \quad \Gamma^{2 n+2} \quad \stackrel{D_{2 n+1}}{\longleftarrow} \quad \Gamma^{2 n+3} \\
& \downarrow \tau_{2 n-1} \quad \downarrow \tau_{2 n} \quad \text { and } \quad \downarrow \tau_{2 n} \quad \downarrow \tau_{2 n+1} \\
& \Gamma^{2 n+1} \stackrel{\cdot D_{2 n}}{\longleftarrow} \quad \Gamma^{2 n+2} \quad \Gamma^{2 n+2} \quad \stackrel{D_{2 n+1}}{\longleftarrow} \quad \Gamma^{2 n+3}
\end{aligned}
$$

Hence, the mapping $\tau$ is a chain map. The fact that it is invertible is immediate from the definition.

\section{The action of the Levi COMPlement}

Recall that the Levi complement is generated by the automorphism $\beta$ with $\beta(a)=b$ and $\beta(b)=a^{-1}$. Let $\nabla_{a}:=\sum_{i=0}^{n-2} a^{i}$. Define

$$
P:=\left(\begin{array}{cc}
0 & \nabla_{a} \\
1 & 0
\end{array}\right) \text { and } Q:=\left(\begin{array}{cc}
0 & -\nabla_{a} \\
1 & 0
\end{array}\right) \text {. }
$$


Lemma 3. The matrices

$$
\left(T_{2 m}^{\prime}\right)_{k, l}=\left\{\begin{array}{cc}
(n-1)^{m+\frac{1-k}{2}} & \text { if } l+k=2 m+2 \text { and } k \text { odd }, \\
-(n-1)^{m-\frac{k}{2}} \cdot \nabla_{a} & \text { if } l+k=2 m+2 \text { and } k \text { even, } \\
0 & \text { else, }
\end{array}\right.
$$

and

$$
\left(T_{2 m-1}^{\prime}\right)_{k, l}=\left\{\begin{array}{cc}
(n-1)^{m+\frac{1-k}{2}} \cdot \nabla_{a} & \text { if } l+k=2 m+2 \text { and } k \text { odd }, \\
(n-1)^{m-\frac{k}{2}} & \text { if } l+k=2 m+2 \text { and } k \text { even }, \\
0 & \text { else, }
\end{array}\right.
$$

satisfy the relations $T_{m+1}^{\prime} D_{m}=\beta\left(D_{m}\right) T_{m}^{\prime}$.

Proof. It is obvious that $\left(T_{2 m}^{\prime} D_{2 m-1}\right)_{i, j}=0$ if $i+j \notin\{2 m+2,2 m+1\}$.

Now assume that $i+j \in\{2 m+2,2 m+1\}$. Then

$$
\left(T_{2 m}^{\prime} D_{2 m-1}\right)_{i, j}=\left\{\begin{array}{cl}
(n-1)^{m+\frac{1-i}{2}}\left(D_{2 m-1}\right)_{2 m+2-i, j} & \text { if } i \text { is odd, } \\
-(n-1)^{m+\frac{-i}{2}}\left(D_{2 m-1}\right)_{2 m+2-i, j} \cdot \nabla_{a} & \text { if } i \text { is even. }
\end{array}\right.
$$

Moreover,

$$
\left(\beta\left(D_{2 m-1}\right) T_{2 m-1}^{\prime}\right)_{i, j}=\left\{\begin{array}{cl}
(n-1)^{\frac{j-1}{2}} \beta\left(D_{2 m-1}\right)_{i, 2 m+1-j} & \text { if } j \text { is odd } \\
(n-1)^{\frac{j-2}{2}} \beta\left(D_{2 m-1}\right)_{i, 2 m+1-j} \cdot \nabla_{a} & \text { if } j \text { is even. }
\end{array}\right.
$$

This is zero if $i+j \notin\{2 m+2,2 m+1\}$. Hence,

$$
\begin{aligned}
& \left(\beta\left(D_{2 m-1}\right) T_{2 m-1}^{\prime}\right)_{i, j} \\
& (n-1)^{\frac{j-1}{2}} \cdot\left\{\begin{array}{cl}
\Delta_{a} & \text { if } i \text { odd and } i+j=2 m+1, \\
a^{-1}-1 & \text { if } i \text { even and } i+j=2 m+1, \\
\Delta_{b} & \text { if } i \text { odd and } i+j=2 m+2, \\
-B & \text { if } i \text { even and } i+j=2 m+2,
\end{array} \quad \text { if } j\right. \text { is odd, } \\
& (n-1)^{\frac{j-2}{2}} \nabla_{a} \cdot\left\{\begin{array}{cl}
\Delta_{a} & \text { if } i \text { odd and } i+j=2 m+1, \\
a^{-1}-1 & \text { if } i \text { even and } i+j=2 m+1, \\
\Delta_{b} & \text { if } i \text { odd and } i+j=2 m+2, \\
-B & \text { if } i \text { even and } i+j=2 m+2,
\end{array} \text { if } j\right. \text { is even. }
\end{aligned}
$$

Of course, the first, fourth, sixth and seventh cases do not occur.

On the other hand,

$$
\begin{aligned}
& \left(T_{2 m}^{\prime} D_{2 m-1}\right)_{i, j} \\
& (n-1)^{m+\frac{1-i}{2}} \cdot\left\{\begin{array}{c}
\Delta_{b} \quad \text { if } j \text { odd and } i+j=2 m+2, \\
B \quad \text { if } j \text { even and } i+j=2 m+2, \\
-A \quad \text { if } j \text { odd and } i+j=2 m+1, \\
\Delta_{a} \quad \text { if } j \text { even and } i+j=2 m+1,
\end{array} \quad \text { if } i\right. \text { is odd, } \\
& -(n-1)^{m-\frac{i}{2}} \nabla_{a} \cdot\left\{\begin{array}{cc}
\Delta_{b} & \text { if } j \text { odd and } i+j=2 m+2, \\
B & \text { if } j \text { even and } i+j=2 m+2, \\
-A & \text { if } j \text { odd and } i+j=2 m+1, \\
\Delta_{a} & \text { if } j \text { even and } i+j=2 m+1,
\end{array}\right.
\end{aligned}
$$

The second, third, fifth and eighth cases do not occur. Using $\nabla_{a} \cdot A=a^{-1}-1$ and $\nabla_{a} \Delta_{a}=(n-1) \Delta_{a}$, one sees that $T_{2 m}^{\prime} D_{2 m-1}=\beta\left(D_{2 m-1}\right) T_{2 m-1}^{\prime}$. 
One has to verify the second of the two equations. The reader will immediately see that this is completely analogous to the above calculations.

Hence, we have proved the proposition.

As in Lemma 2 we get

Lemma 4. $\tau_{k}(x):=\beta(x) T_{k+1}^{\prime}$ for any $k \in \mathbb{N}$ defines a chain isomorphism $P \longrightarrow$ ${ }^{\beta} P$.

\section{Determination OF THE COHOMOlogy GROuP AND THE ACTION OF THE SPECIAL LINEAR GROUP}

The Künneth formula gives a description of $H^{*}\left(C_{n}^{2}, R\right)$ in terms of $H^{*}\left(C_{n}, R\right)$ and certain extension groups. If $R$ is a field, this would have answered our problem immediately; however, for arbitrary $R$ these extension terms make the Künneth formula non-functorial. To determine $H^{*}\left(C_{n}^{2}, R\right)$ and the action of $S L_{2}(\mathbb{Z})$ on it, we proceed via our projective resolution

$$
0 \longleftarrow \Gamma \stackrel{d_{1}}{\longleftarrow} \Gamma^{2} \stackrel{d_{2}}{\longleftarrow} \Gamma^{3} \stackrel{d_{3}}{\longleftarrow} \Gamma^{4} \stackrel{d_{4}}{\longleftarrow} \ldots
$$

and apply $\operatorname{Hom}_{\mathbb{Z} C_{n}^{2}}(-, R)$. This gives a complex

$$
0 \longrightarrow R \stackrel{d_{1}^{*}}{\longrightarrow} R^{2} \stackrel{d_{2}^{*}}{\longrightarrow} R^{3} \stackrel{d_{3}^{*}}{\longrightarrow} R^{4} \stackrel{d_{4}^{*}}{\longrightarrow} \ldots
$$

whose cohomology is $H^{*}\left(C_{n}^{2}, R\right)$.

\section{Lemma 5.}

$$
\left(d_{2 m+1}^{*}\right)_{i, j}=\left\{\begin{aligned}
0 & \text { if } j \text { is odd, } \\
\text { multiplication by } n & \text { if } j \text { is even and } j=i, \\
\text { multiplication by }-n & \text { if } j \text { is even and } j=i-1, \\
0 & \text { otherwise, }
\end{aligned}\right.
$$

and

$$
\left(d_{2 m}^{*}\right)_{i, j}= \begin{cases}\text { multiplication by } n & \begin{array}{l}
\text { if } j \text { is odd and } j=i \\
\text { or } j \text { is even and } j=i+1 \\
\text { otherwise. }
\end{array}\end{cases}
$$

Proof. Observe that the mapping $\gamma \longrightarrow X \gamma \longrightarrow \phi(X \gamma)$ for any $\phi: \Gamma^{k} \longrightarrow R$ with $R$ having trivial $C_{n}^{2}$-action is 0 for $X \in\{A, B\}$. Analogously, the mapping $\gamma \longrightarrow X \gamma \longrightarrow \phi(X \gamma)$ for any $\phi: \Gamma^{k} \longrightarrow R$ with $R$ having trivial $C_{n}^{2}$-action is $n$. for $X \in\left\{\Delta_{a}, \Delta_{b}\right\}$. The result then immediately follows from the structure of the differentials $d$ as determined at the beginning of section 1 .

Now,

$$
\begin{aligned}
\operatorname{ker}\left(d_{2 m+1}^{*}\right) & =R \oplus{ }_{n} R \oplus R \oplus{ }_{n} R \oplus R \oplus \cdots \oplus{ }_{n} R \oplus R \\
& =\left\{\left(g_{1}, g_{2}, \ldots, g_{2 m+1}\right) \in R^{2 m+1} \mid g_{2 i} \in{ }_{n} R, \quad 1 \leq i \leq m\right\}
\end{aligned}
$$

where ${ }_{n} R:=\{r \in R \mid n \cdot r=0\}$, and

$$
\begin{aligned}
i m\left(d_{2 m}^{*}\right) & =n R \oplus 0 \oplus n R \oplus 0 \oplus n R \oplus 0 \oplus \cdots \oplus n R \oplus 0 \oplus n R \\
& =\left\{\left(g_{1}, g_{2}, \ldots, g_{2 m+1}\right) \in R^{2 m+1} \mid g_{2 i}=0 \text { and } g_{2 i+1} \in n R\right\}
\end{aligned}
$$


where $n R=\{n r \in R \mid r \in R\}$. Also,

$$
\begin{aligned}
& \operatorname{ker}\left(d_{2 m}^{*}\right)=\left\{\left(g_{1}, g_{2}, \ldots, g_{2 m}\right) \in R^{2 m} \mid n \cdot\left(g_{2 i-1}+g_{2 i-2}\right)=0\right. \\
& \text { and } \left.n g_{1}=n g_{2 m}=0,2 \leq i \leq m\right\} \\
& =\left\{\left(g_{1}, g_{2}, \ldots, g_{2 m}\right) \in R^{2 m} \mid\left(g_{2 i-1}+g_{2 i-2}\right) \in{ }_{n} R, 2 \leq i \leq m\right. \\
& \text { and } \left.g_{1}, g_{2 m} \in{ }_{n} R\right\} \text {. }
\end{aligned}
$$

Finally,

$$
\begin{aligned}
i m\left(d_{2 m-1}^{*}\right)= & \left\{\left(g_{1}, g_{2}, \ldots, g_{2 m}\right) \in R^{2 m} \mid g_{2 i+1}=-n f_{2 i} \text { and } g_{2 i}=n f_{2 i}\right. \\
& \text { and } \left.g_{1}=g_{2 m}=0 \text { for certain } f_{2 i} \in R \text { and } 1 \leq i \leq m-1\right\} .
\end{aligned}
$$

Using the above we can show the following.

Lemma 6. The groups $H^{*}\left(C_{n}^{2}, R\right)$ are as follows:

$$
H^{2 m}\left(C_{n}^{2}, R\right)=R / n R \oplus{ }_{n} R \oplus R / n R \oplus{ }_{n} R \oplus R / n R \oplus \cdots \oplus{ }_{n} R \oplus R / n R
$$

with $2 m+1$ direct summands and

$$
H^{2 m-1}\left(C_{n}^{2}, R\right) \simeq{ }_{n} R \oplus\left[\bigoplus_{i=1}^{m-1}\left(R / n R \oplus_{n} R\right)\right] \oplus_{n} R
$$

where the summands $R / n R \oplus{ }_{n} R$ in the middle are identified with

$$
\left\{(f+x,-f) \mid f \in R ; x \in_{n} R\right\} /\{(n f,-n f) \mid f \in R\},
$$

the latter of which is in the basis used for describing the matrix $T_{2 m}$.

Proof. The description for the even degrees follows immediately from the description of $\operatorname{ker}\left(d_{2 m+1}^{*}\right)$ and $i m\left(d_{2 m}\right)$.

The same applies for the odd degrees using the identification

$$
\begin{aligned}
R / n R \oplus_{n} R \longleftarrow & \left\{\left(g_{2 i}, g_{2 i+1}\right) \mid g_{2 i}+g_{2 i+1} \in{ }_{n} R\right\} \\
& /\left\{\left(n g_{2 i},-n g_{2 i}\right) \mid g_{2 i} \in R\right\} \\
\left(g_{2 i}+n R, g_{2 i}+g_{2 i+1}\right) \longleftarrow & \left(g_{2 i}, g_{2 i+1}\right)+\left\{\left(n g_{2 i},-n g_{2 i}\right) \mid g_{2 i} \in R\right\} .
\end{aligned}
$$

The fact that this mapping is well defined is immediate. This proves the lemma.

With the preceding lemma we are able to trace back the action of $\alpha$ and $\beta$ on the cohomology groups. Define $\sigma:=\sum_{j=1}^{n-1} j=(n \cdot(n-1)) / 2$.

Lemma 7 . In the above basis, regarding the $2 m \times 2 m$ matrix $\alpha_{2 m}^{*}$ as an $m+1 \times m+1$ matrix of matrices, we get

$$
\left(\alpha_{2 m}^{*}\right)_{i, j}=\left\{\begin{array}{cc}
\text { if } i>j, \\
\left(\begin{array}{cc}
j-1 \\
i-1
\end{array}\right) \cdot\left(\begin{array}{cc}
1 & -\sigma \\
0 & 1
\end{array}\right) & \text { if } i \leq j \leq m, \\
\left(\begin{array}{c}
m \\
i-1
\end{array}\right)_{1}\left(\begin{array}{c}
1 \\
0
\end{array}\right) & \text { if } i<j=m+1, \\
\text { if } i=j=m+1 .
\end{array}\right.
$$

Here,

$$
\left(\begin{array}{cc}
1 & -\sigma \\
0 & 1
\end{array}\right) \cdot\left(\begin{array}{l}
\bar{x} \\
y
\end{array}\right):=\left(\begin{array}{c}
\overline{x-\sigma y} \\
y
\end{array}\right)
$$


Moreover, in the same basis,

$$
\left(\beta_{2 m}^{*}\right)_{i, j}=\left\{\begin{array}{cc} 
& \text { if } i+j \neq m+2, \\
\left(\begin{array}{cc}
0 & -(n-1)^{i} \\
(n-1)^{i-1} & 0
\end{array}\right) & \begin{array}{c}
\text { if } i+j=m+2 \text { and } j \leq m, \\
(n-1)^{m}
\end{array} \\
\text { if } j=m+1 \text { and } i=1 .
\end{array}\right.
$$

Proof. This follows from the description of $\tau_{2 m}$. Since the module $R$ is trivial, the action of $\alpha$ on the free elements does not appear and what remains is the multiplication by $T_{2 m}$. This, however, is realized by the above matrix since multiplication by $\delta$ acts on a trivial module as multiplication by $\sigma$.

\section{Proof of the Theorem}

We are now able to prove Theorem 1. The theorem follows essentially from Lemma 7 We view the symmetric algebra as a polynomial algebra in two variables $X$ and $Y$.

Even degree cohomology: Both generators of the group $S L_{2}(\mathbb{Z})$ fix the $R$ submodule consisting of the odd numbered columns. Hence, this is an $R S L_{2}(\mathbb{Z})$ submodule. For even degree cohomology $H^{2 m}\left(C_{n}^{2}, R\right)$ the module monomorphism of $S_{m}(R / n R)$ into $H^{2 m}\left(C_{n} \times C_{n}, R\right)$ is as follows: $X^{i} Y^{m-i}$ is mapped to the $2 i+1$ column vector (for any $0 \leq i \leq m$ ). Forming the quotient by this submodule, we are left with the set of even numbered columns and with matrices acting as if one had erased odd numbered columns and rows. By the same argument we see that this module is isomorphic to the degree $m-1$ homogeneous polynomials in 2 variables with coefficients in ${ }_{n} R$. Hence the quotient is isomorphic to ${ }_{n} R \cdot S_{m-1}(R)$.

We discuss when the sequence splits. For this, by elementary linear algebra, it is sufficient to have $\sigma \cdot{ }_{n} R \subseteq n R$. Now, $\sigma=n(n-1) / 2$. If $n$ is odd, then $\sigma$ is a multiple of $n$ and thus clearly $\sigma \cdot R \subseteq n R$. It is then easily verified that it is sufficient to deal with the case $n=2^{\nu}$.

In order for the following sequence

$$
0 \longrightarrow S_{m}(R / n R) \longrightarrow H^{2 m}\left(C_{n}^{2}, R\right) \longrightarrow{ }_{n} R \cdot S_{m-1}(R) \longrightarrow 0
$$

to split it is necessary and sufficient to find

$$
\begin{gathered}
\gamma_{1} \in \operatorname{End}_{R}\left(S_{m}(R / n R)\right), \gamma_{4} \in \operatorname{End}_{R}\left({ }_{n} R S_{m-1}(R)\right), \\
\gamma_{2} \in \operatorname{Hom}_{R}\left({ }_{n} R S_{m}(R), S_{m}(R / n R)\right) \text { and } \gamma_{3} \in \operatorname{Hom}_{R}\left(S_{m}(R / n R){ }_{n} R S_{m-1}(R)\right),
\end{gathered}
$$

so that the mapping

$$
\left(\begin{array}{ll}
\gamma_{1} & \gamma_{2} \\
\gamma_{3} & \gamma_{4}
\end{array}\right): H^{2 m}\left(C_{n}^{2}, R\right) \longrightarrow S_{m}(R / n R) \oplus{ }_{n} R \cdot S_{m-1}(R)
$$

is $S L_{2}(\mathbb{Z})$ linear. The latter is equivalent with

$$
\begin{aligned}
& \alpha \gamma_{1}-\gamma_{1} \alpha=\sigma \iota \alpha \gamma_{3} ; \alpha \gamma_{3}=\gamma_{3} \alpha ; \alpha \gamma_{4}=\gamma_{4} \alpha ; \beta \gamma_{1}=\gamma_{1} \beta, \\
& \alpha \gamma_{2}-\gamma_{2} \alpha=\sigma \iota \alpha \gamma_{4} ; \beta \gamma_{2}=\gamma_{2} \beta ; \beta \gamma_{3}=\gamma_{3} \beta ; \beta \gamma_{4}=\gamma_{4} \beta .
\end{aligned}
$$

Here $\iota$ denotes the multiplication by $X$. It is clear that the equations for $\gamma_{2}$ and $\gamma_{4}$ are independent from the equations for $\gamma_{1}$ and $\gamma_{3}$. Moreover, $\gamma_{1}=i d$ and $\gamma_{3}=0$ is a solution for these equations. We may therefore assume that $\gamma_{3}=0$ and $\gamma_{1}=i d$. Since $\gamma_{4}$ is $S L_{2}(\mathbb{Z})$-linear, and since the matrix $\left(\begin{array}{lll}\gamma_{1} & \gamma_{2} \\ \gamma_{3} & \gamma_{4}\end{array}\right)$ is triangular, one might 
modify the above matrix by the endomorphism $\left(\begin{array}{cc}i d & 0 \\ 0 & \gamma_{4}^{-1}\end{array}\right)$ so that one may assume that $\gamma_{4}=i d$ as well.

Summarizing, we look for an $R$-linear mapping $\gamma:{ }_{n} R \cdot S_{m-1}(R) \longrightarrow S_{m}(R / n R)$ satisfying

$$
\alpha \gamma-\gamma \alpha=\sigma \iota \alpha \text { and } \beta \gamma=\gamma \beta
$$

Clearly this implies

$$
\alpha^{i} \gamma-\gamma \alpha^{i}=i \cdot \sigma \iota \alpha^{i}
$$

and thus also

$$
\beta \alpha^{i} \beta^{-1} \gamma-\gamma \beta \alpha^{i} \beta^{-1}=i \cdot \sigma \tilde{\iota} \beta \alpha^{i} \beta^{-1}
$$

for any $i \in \mathbb{N}$. Here we donote $\tilde{\iota}:=\beta \iota \beta^{-1}$. It is now clear that in case $i$ is even, $\gamma=0$ suffices.

We define $\Gamma(2):=\operatorname{ker}\left(P S L_{2}(\mathbb{Z}) \longrightarrow P S L_{2}\left(\mathbb{F}_{2}\right)\right)$ and it is well known that $\Gamma(2)$ is freely generated by $\beta \alpha^{2} \beta^{-1}$ and $\alpha^{2}$.

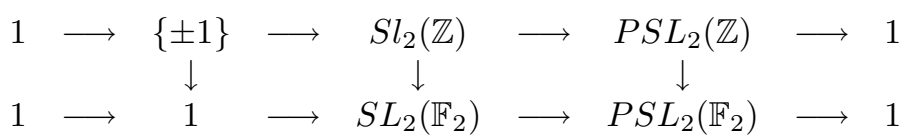

is commutative. All vertical mappings are surjective, hence this induces a central extension

$$
1 \longrightarrow\{ \pm 1\} \longrightarrow \widehat{\Gamma(2)} \longrightarrow \Gamma(2) \longrightarrow 1 \text {. }
$$

Since $\Gamma(2)$ is free on these two matrices and since these two matrices can be realised in $\widehat{\Gamma(2)}$ as well, the sequence is split.

Since for even positive integers $i$ we clearly get that $i \sigma \in n R$, we thus get that the sequence

$$
0 \longrightarrow S_{m}(R / n R) \longrightarrow H^{2 m}\left(C_{n}^{2}, R\right) \longrightarrow{ }_{n} R \cdot S_{m-1}(R) \longrightarrow 0
$$

is split as a sequence of $R \Gamma(2)$-modules. The action of -1 is compatible with the splitting, since -1 is just $\beta^{2}$ and the splitting is compatible with $\beta$. Hence, we conclude that

$$
H^{2 m}\left(C_{n}^{2}, R\right) \simeq S_{m}(R / n R) \oplus{ }_{n} R \cdot S_{m-1}(R)
$$

as $R \widehat{\Gamma(2)}$-modules.

We shall prove that the above sequence is non-split as $R S L_{2}(\mathbb{Z})$-modules if $\left(\begin{array}{c}n \\ 2\end{array}\right) \cdot{ }_{n} R \nsubseteq n R$. By the above, this is equivalent to the existence of a map $\gamma$ so that

$$
\begin{array}{ccc}
{ }_{n} R S_{m-1}(R) & \stackrel{\gamma}{\longrightarrow} & S_{m}(R / n R) \\
\downarrow \cdot\left(\begin{array}{ll}
1 & 1 \\
0 & 1
\end{array}\right) & & \downarrow \cdot\left(\begin{array}{cc}
1 & 1 \\
0 & 1
\end{array}\right) \\
{ }_{n} R S_{m-1}(R) & \stackrel{\gamma+2^{\nu-1} \iota}{\longrightarrow} & S_{m}(R / n R)
\end{array}
$$

is a commutative diagram. Interpreting the symmetric algebra as a polynomial algebra, we have to find coefficients $a_{i} \in(R / n R)$ so that

$$
\gamma\left(Y^{m-1}\right)=\sum_{i=0}^{m} a_{i} X^{i} Y^{m-i}
$$

Now, if we set $g:=\left(\begin{array}{ll}1 & 1 \\ 0 & 1\end{array}\right)$ since $g$ acts trivially on $Y^{m-1}$, we must have

$$
\lambda\left(\gamma\left(Y^{m-1}\right)+2^{\nu-1} \iota\left(Y^{m-1}\right)\right)=\lambda g \gamma\left(Y^{m-1}\right)
$$


which gives the equation

$$
\lambda\left(\sum_{i=1}^{m} \sum_{j=1}^{i} a_{i}\left(\begin{array}{l}
i \\
j
\end{array}\right) X^{i-j} Y^{m-i+j}\right)=\lambda 2^{\nu-1} Y^{m}
$$

for any $\lambda \in{ }_{n} R$. If one expresses this projective equation in affine coordinates cutting out the point in infinity $Y=0$, one gets

$$
\lambda \sum_{i=1}^{m} a_{i}\left(1+\frac{X}{Y}\right)^{i}=2^{\nu-1} \lambda .
$$

But, since $\left\{\left(\frac{X}{Y}+1\right)^{i} \mid i \in \mathbb{N}\right\}$ is a basis for the polynomial ring in one variable over any coefficient ring, this equation has a solution if and only if $\lambda \cdot 2^{\nu-1}=0$ (in $R / n R)$ for any $\lambda \in{ }_{n} R$. This in turn is equivalent to

$$
\left(\begin{array}{l}
n \\
2
\end{array}\right) \cdot{ }_{n} R \subseteq n R .
$$

Odd degree cohomology: The universal coefficient theorem provides us with a short exact sequence

$$
0 \longrightarrow H^{2 m+1}\left(C_{n}^{2}, \mathbb{Z}\right) \otimes_{\mathbb{Z}} R \longrightarrow H^{2 m+1}\left(C_{n}^{2}, R\right) \longrightarrow \operatorname{Tor}_{1}^{\mathbb{Z}}\left(H^{2 m+2}\left(C_{n}^{2}, \mathbb{Z}\right), R\right) \longrightarrow 0
$$

and for integer coefficients we readily see that from the above $H^{2 m}\left(C_{n}^{2}, \mathbb{Z}\right)=$ $S_{m}(\mathbb{Z} / n \mathbb{Z})$ and $H^{2 m+1}\left(C_{n}^{2}, \mathbb{Z}\right) \simeq S_{m-1}(\mathbb{Z} / n \mathbb{Z})$. Of course, this can be seen directly from our description as well. It should be possible to decide upon the splitting question this way, though we did not try to do so here.

Finally we remark that the above implies that $H^{2 k}\left(C_{n}^{2}, \mathbb{Z}\right) \simeq H^{2 k+3}\left(C_{n}^{2}, \mathbb{Z}\right) \simeq$ $S_{k}(\mathbb{Z} / n \mathbb{Z})$ for all $k \geq 2$.

Let $n=p$ be an odd prime. Then, $S t \simeq H^{2 p}\left(C_{p}^{2}, \mathbb{Z}\right) \simeq H^{2 p+3}\left(C_{p}^{2}, \mathbb{Z}\right)$ is the Steinberg module. Using Chapman's result [2] on the cohomology ring $H^{*}(A, \mathbb{Z})$ for an abelian group $A$, one sees that this shift of three degrees essentially comes from the fact that $H^{*}\left(C_{n}^{2}, \mathbb{Z}\right)$ is generated in degree 2 and 3 and the degree 3 cohomology is just the trivial module.

\section{REFERENCES}

1. D. Benson, Representations and Cohomology, Cambridge 1991.

2. G. R. Chapman, The cohomology ring of a finite abelian group, Proc. London Math. Soc. 45 (1982) 564-576. MR 84g:20091

Department of Mathematics, Vrije Universiteit Brussel, Pleinlaan 2, 1050 Brussel, BELGIUM

E-mail address: ef jesper@vub.ac.be

Lamfa, Faculté de Mathématiques, Université de Picardie Jules Verne, 33 rue St Leu, 80039 Amiens Cedex, France

E-mail address: Alexander.Zimmermann@u-picardie.fr 